\title{
Treatment Stacking: Optimizing Therapeutic Regimens for Hidradenitis Suppurativa
}

\author{
Terri Shih, BS; Vivian Shi, MD; Jennifer L. Hsiao, MD
}

$\mid$ idradenitis suppurativa (HS) is a debilitating chronic condition that often is recalcitrant to first-line treatments, and mechanisms underlying its pathology remain unclear. Existing data suggest a multifactorial etiology with different pathophysiologic contributors, including genetic, hormonal, and immune dysregulation factors. At this time, only one medication (adalimumab) is US Food and Drug Administration approved for HS, but multiple medical and procedural therapies are available. ${ }^{1}$ Herein, we discuss the concept of treatment stacking, or the combination of unique therapeutic modalitiesan approach we believe is key to optimizing management of HS patients.

\section{Stacking Treatments for HS}

Unlike psoriasis, in which a single biologic agent may provide $100 \%$ clearance (psoriasis area and severity index 100 [PASI 100]) without adjuvant treatment, ${ }^{2,3}$ the field of HS currently lacks medications that are efficacious to that degree of suecess as monotherapy. In HS, the benchmark for a positive treatment outcome is Hidradenitis Suppurativa Clinical Response 50 (HiSCR50), ${ }^{4}$ a 50\% reduction in inflammatory lesion count-a far less stringent marker for disease improvement. Thus, providers should design HS treatment regimens with a model of combining therapies and shift away from monotherapy. Targeting different pathophysiologic pathways by stacking multiple treatments may provide synergistic benefits for HS patients. Treatment stacking is a familiar concept in acne; for instance, patients who benefit tremendously from isotretinoin may still require a hormone-modulating treatment (eg, spironolactone) to attain optimal results.

Adherence to a rigid treatment algorithm based on disease severity limits the potential to create comprehensive regimens that account for unique patient characteristics and clinical manifestations. When evaluating an HS patient, providers should systematically consider each pathophysiologic factor and target the ones that appear to be most involved in that particular patient. The North American HS guidelines ilutustrate this point by supporting use of several treatments across different Hurley stages, such as recommending hormonal treatment in patients with Hurley stages 1,2 , or $3 .^{1}$ Of note, treatment stacking also includes procedural therapies. Surgeons typically prefer a patient's disease management to be optimized prior to surgery, including reduced drainage and inflammation. In addition, even after surgery, patients often still require medical management to prevent continued disease worsening.

\section{Treatment Pathways for HS}

A multimodal approach with treatment stacking (Figure) can be useful to all HS patients, from those with the mildest to the most severe disease. Modifiable pathophysiologic factors and examples of their targeted treatments include (1) follicular occlusion (eg, oral retinoids), (2) metabolic dysfunction (eg, metformin), (3) hormones (eg, oral contraceptive pills, spironolactone, finasteride), (4) dysbiosis (eg, antibiotics such as clindamycin and rifampin combination therapy), (5) immune dysregulation

\footnotetext{
Ms. Shih and Dr. Hsiao are from the University of California, Los Angeles. Ms. Shih is from the David Geffen School of Medicine, and Dr. Hsiao is from the Division of Dermatology. Dr. Shi is from the Department of Dermatology, University of Arkansas for Medical Sciences, Little Rock. Ms. Shih reports no conflict of interest. Dr. Shi is on the Board of Directors for the Hidradenitis Suppurativa Foundation and is a stock shareholder for Learn Health. Dr. Shi also has served as an advisory board member, investigator, or speaker and/or has received research funding from AbbVie; Boehringer Ingelheim; Burt's Bees, Inc; CQuell/Altus Lab; Dermira, Inc; Eli Lilly and Company; Galderma; Gpskin; Incyte Corporation; Kiniksa Pharmaceuticals; LEO Pharma; Menlo Therapeutics; MyOR; Novartis; Pfizer; Polyfins Technology; Regeneron Pharmaceuticals; Sanofi Genzyme; Skin Actives Scientific; Sun Pharmaceutical Industries Ltd; TARGET PHARMASOLUTIONS; and UCB. Dr. Hsiao is on the Board of Directors for the Hidradenitis Suppurativa Foundation, a speaker for AbbVie, and consultant for Novartis.

Correspondence: Jennifer L. Hsiao, MD, Division of Dermatology, UCLA, 2020 Santa Monica Blvd, Ste 510, Santa Monica, CA 9040 (j.hsiao.publications@gmail.com). doi:10.12788/cutis.0359
} 


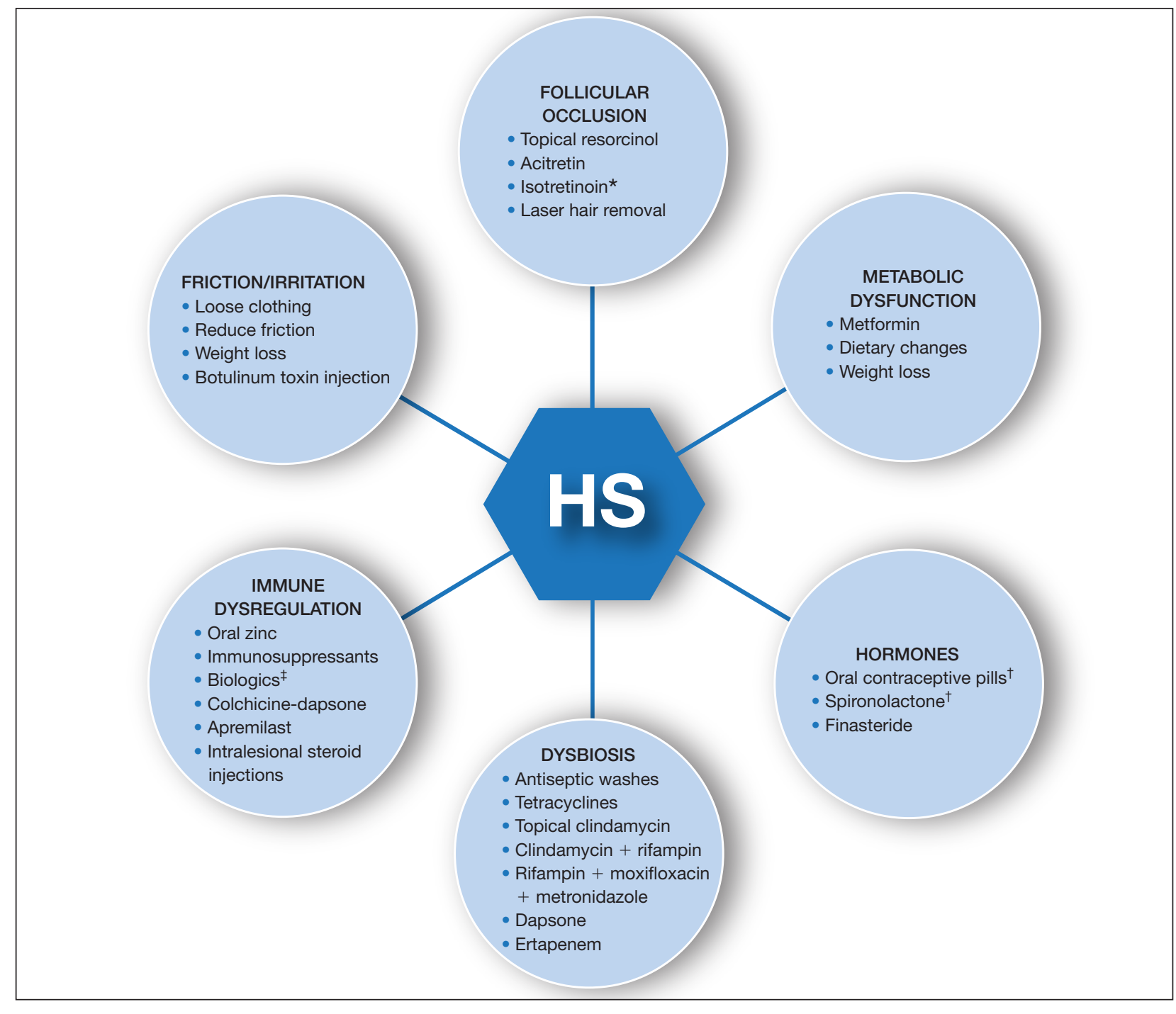

Targeted treatments for modifiable pathophysiologic arms of hidradenitis suppurativa (HS). Surgical and laser excisions (not shown) remove persistent inflamed and diseased tissue. Asterisk indicates mixed data in literature; should be considered in patients with severe acne. Dagger indicates exclusive usage in female HS patients. Double dagger indicates biologics including anti-tumor necrosis factor $\alpha$, IL-1, IL-17, IL-12/23, and IL-23.

(eg, biologic agents), and (6) friction/irritation (eg, weight loss, clothing recommendations).

Combining treatments from different pathways enables potentiation of individual treatment efficacies. A female patient with only a few HS nodules that flare with menses may be well controlled with spironolactone as her only systemic agent; however, she still may benefit from use of an antiseptic wash, topical clindamycin, and lifestyle changes such as weight loss and reduction of mechanical irritation. A patient with severe recalcitrant HS could notably benefit from concomitant biologic, systemic antibiotic, and hormonal/metabolic treatments. If disease control is still inadequate, agents within the same class can be switched (eg, choosing a different biologic) or other disease-modifying agents such as colchicine also can be added. The goal is to create an effective treatment toolbox with therapies targeting different pathophysiologic arms of HS and working together in synergy. Each tool can be refined by modifying dosing frequency and duration of use to strive for optimal response. At this time, the literature on HS combination therapy is sparse. A retrospective study of 31 patients reported promising combinations, including isotretinoin with spironolactone for mild disease, isotretinoin or doxycycline with adalimumab for moderate disease, and cyclosporine with adalimumab for severe disease. ${ }^{5}$ Larger prospective studies on clinical response to different combination regimens are warranted.

\section{Optimizing Therapy for HS and Its Comorbidities}

Additional considerations may further optimize treatment plans. Some therapies benefit all patients; for example, providers should counsel all HS patients on healthy weight management, optimized clothing choices, ${ }^{6}$ and friction reduction in the intertriginous folds. Providers 
also may consider adding therapies with faster onset of efficacy as a bridge to long-term, slower-onset therapies. For instance, female HS patients with menstrual flares who are prescribed spironolactone also may benefit from a course of systemic antibiotics, which typically provides more prompt relief. Treatment regimens also can concomitantly treat HS and its comorbidities. ${ }^{7}$ For example, metformin serves a dual purpose in HS patients with diabetes mellitus, and adalimumab in patients with both HS and inflammatory bowel disease.

\section{Final Thoughts}

The last decade has seen tremendous growth in HS research ${ }^{8}$ coupled with a remarkable expansion in the therapeutic pipeline. ${ }^{9}$ However, currently no single therapy for HS can guarantee satisfactory disease remission or durability of remission. The contrast between clinical trials and real-world practice should be acknowledged; the former often is restrictive in design with monotherapy and allowance of very limited concomitant treatments, such as topical or oral antibiotics. This limits our ability to draw conclusions regarding the additive synergistic potential of different therapeutics in combination. In clinical practice, we are not restricted by monotherapy trial protocols. As we await new tools, treatment stacking allows for creating a framework to best utilize the tools that are available to us.

Although HS has continued to affect the lives many patients, improved understanding of u pathophysiology and a well-placed sense from all stakeholders (ie, patients, clinicians, industry partners) has pushed this field forward. Until

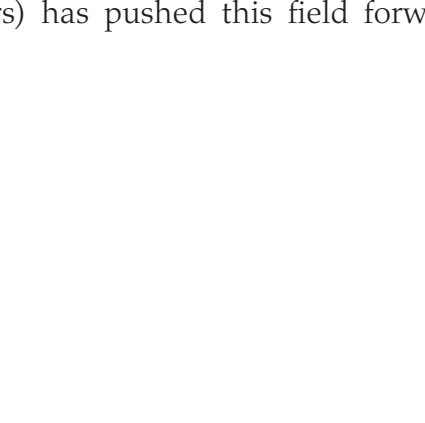

our therapeutic armamentarium has expanded to include highly efficacious monotherapy options, providers should consider treatment stacking for every HS patient.

\section{REFERENCES}

1. Alikhan A, Sayed C, Alavi A, et al. North American clinical management guidelines for hidradenitis suppurativa: a publication from the United States and Canadian Hidradenitis Suppurativa Foundations: part II: topical, intralesional, and systemic medical management. J Am Acad Dermatol. 2019;81:91-101. doi:10.1016/j.jaad.2019.02.068

2. Reich K, Warren RB, Lebwohl M, et al. Bimekizumab versus secukinumab in plaque psoriasis. N Engl J Med. 2021;385:142-152. doi:10.1056/NEJMoa2102383

3. Imafuku S, Nakagawa H, Igarashi A, et al. Long-term efficacy and safety of tildrakizumab in Japanese patients with moderate to severe plaque psoriasis: results from a 5 -year extension of a phase 3 study (reSURFACE 1). J Dermatol. 2021;48:844-852. doi:10.1111 /1346-8138.15763

4. Kimball AB, Okun MM, Williams DA, et al. Two phase 3 trials of adalimumab for hidradenitis suppurativa. NEngl J Med. 2016;375:422-434. doi:10.1056/NEJMoa1504

5. McPhie ML, Bridgman AC, Kirch of MG. Combination therapies for hidradenitis suppurativa: a retrospective chart review of 31 patients. J Cutan Med Surg. 2019;23:270-276. doi:10.1177/1203475418823529

6. Loh TY, Hendricks AJ, Hsiao JL, et al. Undergarment and fabric selection in the management of hidradenitis suppurativa. Dermatol Basel Switz. 2021;237:119-124. doi:10.1159/000501611

7. Garg A, Malviya N, Strunk A, et al. Comorbidity screening in hidradenitis suppurativa: evidence-based recommendations from the US and Canadian Hidradenitis Suppurativa Foundations [published online January 23, 2021]. J Am Acad Dermatol. doi:10.1016/j jaad.2021.01.059

8. Savage KT, Brant EG, Flood KS, et al. Publication trends in hidradenitis suppurativa from 2008 to 2018. J Eur Acad Dermatol Venereol. 2020;34:1885-1889. doi:10.1111/jdv.16213

9. van Straalen KR, Schneider-Burrus S, Prens EP. Current and future treatment of hidradenitis suppurativa. Br J Dermatol. 2020;183:E178-E187. doi:10.1111/bjd.16768 